\title{
REPRESENTATION OF A COMPLETELY BOUNDED BIMODULE MAP
}

\author{
QIYUAN NA
}

(Communicated by Palle E. T. Jorgensen)

\begin{abstract}
In this paper, we give a representation for a completely bounded $A-B$ bimodule map into $B(H)$, where $A$ and $B$ are unital operator subalgebras of $B(H)$. When $A$ and $B$ are $C^{*}$-subalgebras we give a new proof of the Wittstock's theorem by using this representation. We also prove that a von Neumann algebra is an injective operator bimodule over its unital operator algebras if and only if it is a finitely injective operator bimodule.
\end{abstract}

\section{INTRODUCTION}

An operator space is a $L^{\infty}$-matricially normed space (see [12]). A unital operator algebra is an operator space and is also a unital algebra with completely contractive multiplication (see [2]). An operator bimodule over two unital operator algebras is an operator space and is also a unital bimodule with completely contractive multiplication (see [3]). While there is an extensive literature on the representation of completely bounded and related types of linear maps (see [1, $3,7-10]$, and others), there has been relatively little done in the way of representating completely bounded bimodule maps. One notable exception is Smith's representation of completely bounded bimodule maps from $K(H)$ into $B(H)$. This paper shows in particular that $M_{6}$ is not an injective operator bimodule over a pair of unital operator subalgebras of $M_{6}$ (see [14]). We are motivated by this fact to study the representation of completely bounded bimodule maps and the injectivity of $B(H)$ as an operator bimodule.

In $\S 2$, we first give a representation for a completely bounded $A-B$ bimodule map into $B(H)$ when $A$ and $B$ are $C^{*}$-subalgebras of $B(H)$. Using this representation, we give a new proof of Wittstock's theorem. Later, we generalize the representation to the case that $A$ and $B$ are unital operator subalgebras of $B(H)$. In $\S 3$, we prove that a von Neumann algebra is an injective operator bimodule over two unital subalgebras if and only if it is a finitely injective operator bimodule.

Throughout this paper, all subspaces, operator subalgebras, operator subbimodules, etc., are closed. We use the term homomorphism for a bimodule

Received by the editors April 12, 1993 and, in revised form, July 6, 1993.

1991 Mathematics Subject Classification. Primary 47A20; Secondary 46L05.

Key words and phrases. Operator bimodules, representation, dilation, injectivity, finite injectivity, finitely generated operator bimodule. 
map when no confusion may result. An embedding is an injective homomorphism. A homeomorphism is a surjective embedding. We do not distinguish between $Y$ an operator subbimodule of $X$ and a completely isometrical embedded copy of $Y$ in $X$. Every vector space is over the complex numbers, and every map is linear.

Suppose $X$ and $Y$ are $A-B$ operator bimodules over unital operator algebras $A$ and $B$. We denote by $\operatorname{Hom}(X, Y)$ the space of all completely bounded homomorphisms from $X$ into $Y$. If $X$ is a subset of a unital $C^{*}$-algebra, we denote by $C^{*}(X)$ the unital $C^{*}$-algebra generated by $X$.

\section{REPRESENTATION OF A COMPLETELY BOUNDED BIMODULE MAP}

We begin this section with a simple lemma (see [6]).

Lemma 2.1. Suppose that $A$ and $B$ are operator algebras with $1_{A}$ and $1_{B}$, respectively. Then an operator space $X$ is an $A-B$ operator bimodule if and only if there exists a completely contractive trilinear map $\Phi: A \times X \times B \rightarrow X$ that satisfies

$$
\Phi\left(a_{1} a_{2}, x, b_{1} b_{2}\right)=\Phi\left(a_{1}, \Phi\left(a_{2}, x, b_{1}\right), b_{2}\right)
$$

and

$$
\Phi\left(1_{A}, x, 1_{B}\right)=x
$$

for all $a_{1}, a_{2} \in A, b_{1}, b_{2} \in B$, and $x \in X$. Moreover, the multiplication is determined by $\Phi$ via the equation $\Phi(a, x, b)=$ axb for all $a \in A, b \in B$, and $x \in X$.

The following theorem gives us the representations of completely bounded $C^{*}$-bimodule maps.

Theorem 2.2. Suppose that $A$ and $B$ are unital $C^{*}$-subalgebras of $B(H)$, where $H$ is a Hilbert space. Suppose that $X$ is an $A-B$ operator bimodule. Then every completely bounded $A-B$ bimodule map $\phi$ from $X$ into $B(H)$ has a representation $\left(V_{1}, \pi_{1}, \theta, \pi_{2}, V_{2}, K\right)$, where $\pi_{1}$ and $\pi_{2}$ are $*$-representations of $A$ and $B$ on a Hilbert space $K, \theta$ is a complete contraction from $X$ into $B(K)$, and $\mathrm{H}_{\rightarrow} \stackrel{V_{2}}{\rightarrow} \stackrel{V_{1}}{\rightarrow} H$ are bridging maps such that

$$
\begin{gathered}
\phi(x)=V_{1} \theta(x) V_{2} ; \\
\theta(a x b)=\pi_{1}(a) \theta(x) \pi_{2}(b) ; \\
a V_{1}=V_{1} \pi_{1}(a), \quad V_{2} b=\pi_{2}(b) V_{2} ; \\
\|\phi\|_{\mathrm{cb}}=\left\|V_{1}\right\|\left\|V_{2}\right\|
\end{gathered}
$$

for all $a \in A, x \in X$, and $b \in B$.

Proof. Suppose that $\left(\tilde{\pi}_{1}, \tilde{\theta}, \tilde{\pi}_{2}, \widetilde{K}\right)$ is a representation of $X$ in Corollary 3.3 of [3], i.e., $\tilde{\pi}_{1}$ and $\tilde{\pi}_{2}$ are *-representations of $A$ and $B$ on a Hilbert space $\widetilde{K}$ and $\tilde{\theta}: X \rightarrow B(\widetilde{K})$ is a complete isometry such that

$$
\tilde{\theta}(a x b)=\tilde{\pi}_{1}(a) \tilde{\theta}(x) \tilde{\pi}_{2}(b)
$$

for all $a \in A, x \in X$, and $b \in B$. Applying Lemma 2.1 above, we see that $\tilde{\theta}(X)$ is an $A-B$ operator bimodule with the bimodule multiplication given by $a y b=\tilde{\pi}_{1}(a) y \tilde{\pi}_{2}(b)$ for all $a \in A, Y \in \tilde{\theta}(X)$, and $b \in B$. Moreover, $\bar{\phi}=\phi \circ \tilde{\theta}^{-1}$ is a completely bounded $A-B$ bimodule map from $\tilde{\theta}(X)$ into 
$B(H)$. Therefore, there exists a $*$-representation $\pi$ of $B(\tilde{K})$ on some Hilbert space $K$ and bridging maps $H \stackrel{\widetilde{V}_{2}}{\rightarrow} K \stackrel{\widetilde{V}_{1}}{\rightarrow} H$ such that

$$
\bar{\phi}(y)=\widetilde{V}_{1} \pi(y) \tilde{V}_{2}
$$

for all $y \in \tilde{\theta}(X)$ and $\|\phi\|_{\mathrm{cb}}=\left\|\tilde{V}_{1}\right\|\left\|\tilde{V}_{2}\right\|$ (see [7]). Since for each $x \in X$,

$$
\phi(x)=\bar{\phi}(\theta(x))=\widetilde{V}_{1} \pi\left(\tilde{\pi}_{1}(\mathbf{1})\right) \pi(\tilde{\theta}(x)) \pi\left(\tilde{\pi}_{2}(\mathbf{1})\right) \tilde{V}_{2},
$$

we may assume that $\widetilde{V}_{1}=\widetilde{V}_{1} \pi\left(\tilde{\pi}_{1}(\mathbf{1})\right), \widetilde{V}_{2}=\pi\left(\tilde{\pi}_{2}(\mathbf{1})\right) \tilde{V}_{2}$, where 1 is the unit of $B(H)$. Then

Let $P: K \rightarrow\left[\pi(\tilde{\theta}(X)) \widetilde{V}_{2} H\right]$ be the orthogonal projection onto $\left[\pi(\tilde{\theta}(X)) \widetilde{V}_{2} H\right]$.

$$
\bar{\phi}=\widetilde{V}_{1} \pi \widetilde{V}_{2}=\widetilde{V}_{1} P \pi \widetilde{V}_{2} .
$$

Since $\pi\left(\tilde{\pi}_{1}(a)\right) \pi(\tilde{\theta}(x))=\pi(\tilde{\theta}(a x))$ for all $a \in A$ and $x \in X$, we have $P \in$ $\pi\left(\tilde{\pi}_{1}(A)\right)^{\prime}$, the commutant of $\pi\left(\tilde{\pi}_{1}(A)\right)$. Moreover, since for each $a \in A$ and $x \in X$,

$$
\widetilde{V}_{1} \pi\left(\tilde{\pi}_{1}(a)\right) \pi(\tilde{\theta}(x)) \widetilde{V}_{2}=a \widetilde{V}_{1} \pi(\tilde{\theta}(x)) \widetilde{V}_{2},
$$

we have

$$
\widetilde{V}_{1} \pi\left(\tilde{\pi}_{1}(a)\right) P=a \widetilde{V}_{1} P
$$

for all $a \in A$. Let $Q: K \rightarrow\left[\pi(\tilde{\theta}(X))^{*} P \widetilde{V}_{1}^{*} H\right]$ be the orthogonal projection onto $\left[\pi(\tilde{\theta}(X))^{*} P \widetilde{V}_{1}^{*} H\right]$. Since $P \in \pi\left(\tilde{\pi}_{1}(A)\right)^{\prime}$ and

$$
\widetilde{V}_{1} P \pi(\tilde{\theta}(x)) \widetilde{V}_{2} b=\widetilde{V}_{1} P \pi(\tilde{\theta}(x)) \pi\left(\tilde{\pi}_{2}(b)\right) \tilde{V}_{2}
$$

for all $x \in X$ and $b \in B$, we have

$$
b^{*} \widetilde{V}_{2}^{*} Q=\widetilde{V}_{2}^{*} \pi\left(\tilde{\pi}_{2}(b)\right)^{*} Q
$$

by taking adjoints. Thus,

$$
Q \widetilde{V}_{2} b=Q \pi\left(\tilde{\pi}_{2}(b)\right) \tilde{V}_{2}
$$

for all $b \in B$. Since

$$
\pi\left(\tilde{\pi}_{2}(b)\right)^{*} \pi(\tilde{\theta}(x))^{*} P \widetilde{V}_{1}^{*} h=\pi(\tilde{\theta}(x b))^{*} P \widetilde{V}_{1}^{*} h
$$

for all $b \in B, x \in X$, and $h \in H$, we have $Q \in \pi\left(\tilde{\pi}_{2}(B)\right)^{\prime}$. For any $x \in X$, $h_{1}, h_{2} \in H$

$$
\begin{aligned}
\left(\phi(x) h_{1}, h_{2}\right) & =\left(\widetilde{V}_{1} P \pi(\tilde{\theta}(x)) \widetilde{V}_{2} h_{1}, h_{2}\right) \\
& =\left(\widetilde{V}_{2} h_{1}, \pi(\tilde{\theta}(x))^{*} P \widetilde{V}_{1}^{*} h_{2}\right) \\
& =\left(\widetilde{V}_{2} h_{1}, Q \pi(\tilde{\theta}(x))^{*} P \widetilde{V}_{1}^{*} h_{2}\right) \\
& =\left(\widetilde{V}_{1} P \pi(\tilde{\theta}(x)) Q \widetilde{V}_{2} h_{1}, h_{2}\right) .
\end{aligned}
$$

Therefore,

$$
\phi(x)=\bar{\phi}(\tilde{\theta}(x))=\widetilde{V}_{1} P \pi(\tilde{\theta}(x)) Q \widetilde{V}_{2} .
$$

Now setting $V_{1}=\widetilde{V}_{1} P, \quad V_{2}=Q \widetilde{V}_{2}, \pi_{1}=\pi \circ \tilde{\pi}_{1}, \pi_{2}=\pi \circ \tilde{\pi}_{2}$, and $\theta=$ $P(\pi \circ \tilde{\theta}) Q$, we obtain the representation $\left(V_{1}, \pi_{1}, \theta, \pi_{2}, V_{2}, K\right)$ with the properties claimed in the theorem. 
Remark 2.1. The representation in Theorem 2.2 depends on the representation of the $A-B$ operator bimodule $X$. We will use this to give a new and totally different approach to the proof of Wittstock's theorem (cf. $[15,5]$ ).

Suppose that $A$ and $B$ are unital operator algebras, and suppose that $X$ is an $A-B$ operator bimodule. Recall that $X$ is an injective $A-B$ operator bimodule if for each $A-B$ operator submimodule $Y_{1}$ of an $A-B$ operator bimodule $Y$ and each completely bounded homomorphism $\phi: Y_{1} \rightarrow X$ there exists a completely bounded homomorphism $\tilde{\phi}: Y \rightarrow X$ which extends $\phi$ and has the same cb-norm. In other words, $X$ is an injective object in the category of $A-B$ operator bimodules and completely bounded homomorphisms (see [5]).

Theorem 2.3. Suppose that $A$ and $B$ are unital $C^{*}$-subalgebras of $B(H)$, where $H$ is a Hilbert space. Then $B(H)$ is an injective $A-B$ operator bimodule.

Proof. Suppose that $X$ is an $A-B$ operator subbimodule of an $A-B$ operator bimodule $Y$. Suppose $\phi \in \operatorname{Hom}(X, B(H))$. Suppose that $\left(\tilde{\pi}_{1}, \tilde{\theta}, \tilde{\pi}_{2}, \tilde{K}\right)$ is a representation of $Y$. Then $\left(\tilde{\pi}_{1},\left.\tilde{\theta}\right|_{X}, \tilde{\pi}_{2}, \tilde{K}\right)$ is a representation of $X$. By using the notation in the proof of Theorem 2.2, $\phi$ has a representation $\left(V_{1}, \pi_{1}, \hat{\theta}, \pi_{2}, V_{2}, K\right)$ with the properties described there, where $\hat{\theta}=$ $P\left(\left.\pi \circ \tilde{\theta}\right|_{X}\right) Q$. Now if we replace $\hat{\theta}$ by $\theta=P(\pi \circ \theta) Q$, then it is easy to see that $\left(V_{1}, \pi_{1},\left.\theta\right|_{X}, \pi_{2}, V_{2}, K\right)$ is a representation of $\phi$ with the properties claimed in Theorem 2.2. Moreover,

$$
\begin{aligned}
\theta(a y b) & =P \pi\left(\tilde{\pi}_{1}(a)\right) \pi(\tilde{\theta}(y)) \pi\left(\tilde{\pi}_{2}(b)\right) Q \\
& =\pi_{1}(a) \theta(y) \pi_{2}(b)
\end{aligned}
$$

for all $a \in A, y \in Y$, and $b \in B$. Let $\tilde{\phi}: Y \rightarrow B(H)$ be given by the representation $\left(V_{1}, \pi_{1}, \theta, \pi_{2}, V_{2}, K\right)$; i.e., let $\tilde{\phi}=V_{1} \theta V_{2}$. Then $\tilde{\phi} \in \operatorname{Hom}(Y, B(H))$, extends $\phi$, and has the same cb-norm $\|\phi\|_{\mathrm{cb}}$.

When $A$ and $B$ are unital operator algebras, we still have the same form representation for a completely bounded $A-B$ bimodule map as we do in the case $A-B$ are unital $C^{*}$-algebras. However, the representation tells less information than it does in the latter case.

Corollary 2.4. Suppose that $A$ and $B$ are unital operator algebras of $B(H)$, where $H$ is Hilbert space. Suppose that $X$ is an $A-B$ operator bimodule. Then every completely bounded $A-B$ bimodule map $\phi$ from $X$ into $B(H)$ has representation $\left(V_{1}, \pi_{1}, \theta, \pi_{2}, V_{2}, K\right)$, where $\pi_{1}$ and $\pi_{2}$ are *-representation of $C^{*}(A)$ and $C^{*}(B)$ on a Hilbert space $K, \theta$ is a complete contraction from $X$ into $B(K)$, and $H \stackrel{V_{2}}{\rightarrow} K \stackrel{V_{1}}{\rightarrow} H$ are bridging maps such that

$$
\begin{gathered}
\phi(x)=V_{1} \theta(x) V_{2} ; \\
\theta(a x b)=\pi_{1}(a) \theta(x) \pi_{2}(b) ; \\
a V_{1}=V_{1} \pi_{1}(a), \quad V_{2} b=\pi_{2}(b) V_{2} ; \\
\|\phi\|_{\mathrm{cb}}=\left\|V_{1}\right\|\left\|V_{2}\right\|
\end{gathered}
$$

for all $a \in A, x \in X$, and $b \in B$.

Proof. By a theorem in [6], there exists a completely bounded $C^{*}(A)-C^{*}(B)$ bimodule map $\tilde{\phi}: \tilde{X} \rightarrow B(H)$ such that $\phi=\tilde{\phi} \circ \alpha$ and $\|\phi\|_{\mathrm{cb}}=\|\tilde{\phi}\|_{\mathrm{cb}}$, where $\tilde{X}$ 
is a dilation of $X$ which is a $C^{*}(A)-C^{*}(B)$ operator bimodule and $\alpha: X \rightarrow \tilde{X}$ is a complete contractive $A-B$ bimodule map. Applying Theorem 2.2 to $\tilde{\phi}$ and then restricting to $X$, we get the representation for $\phi$.

Remark 2.2. It is easy to see that the representation in Corollary 2.4 depends on the dilation $\widetilde{X}$ of $X$. We may not use Corollary 2.4 to get an analogous result of Theorem 2.3 when $A$ and $B$ are unital operator algebras. The reason is that when $X$ is an $A-B$ operator sub-bimodule of an $A-B$ operator bimodule $Y$, the dilation $\widetilde{X}$ is not necessarily a $C^{*}(A)-C^{*}(B)$ operator subbimodule of the dilation $\tilde{Y}$. In fact, $M_{6}$ is not an $A-B$ operator bimodule for some unital operator subalgebras $A$ and $B$ of $M_{6}$ (see [14]). The following section will give a sufficient and necessary condition for $B(H)$ to be an injective $A-B$ operator bimodule for unital operator subalgebras $A$ and $B$ of $B(H)$.

\section{INJECTIVITY OF OPERATOR BIMODULES}

We say that an $A-B$ operator bimodule is finitely generated if there exists a finite subset $F$ of $X$ such that $X=[A F B]$. The concept defined in the following definition seems to be a weaker notion than injectivity.

Definition 3.1. An $A-B$ operator bimodule $X$ is called a finitely injective $A-B$ operator bimodule if for any two finitely generated $A-B$ operator bimodules $X_{1}$ and $X_{2}$ where $X_{1}$ is an $A-B$ operator subbimodule of $X_{2}$ and any $\phi \in$ $\operatorname{Hom}\left(X_{1}, X\right)$ there is a $\tilde{\phi} \in \operatorname{Hom}\left(X_{2}, X\right)$ which extends $\phi$ and has the same cb-norm. Roughly speaking, $X$ is an injective object in the category of finitely generated $A-B$ operator bimodules and completely bounded homomorphism.

The following theorem shows that injectivity and finite injectivity of operator bimodules are the same for von Neumann algebras. It should provide a useful tool to deal with the injectivity question for operator bimodules.

Theorem 3.1. Suppose that $\mathscr{D}$ is a von Neumann algebra. Suppose that $A$ and $B$ are unital operator subalgebras of $\mathscr{D}$. Then $\mathscr{D}$ is an injective $A-B$ operator bimodule if and only if $\mathscr{D}$ is a finitely injective $A-B$ operator bimodule.

Proof. It is obvious that injectivity implies finite injectivity. Suppose $\mathscr{D}$ is a finitely injective $A-B$ operator bimodule. We prove $\mathscr{D}$ is an injective $A-B$ operator bimodule. Suppose that $X_{1}$ is an $A-B$ operator subbimodule of an $A-B$ operator bimodule $X_{2}$ and $\phi \in \operatorname{Hom}\left(X_{1}, \mathscr{D}\right)$. Without loss of generality, we may assume that $\|\phi\|_{\mathrm{cb}}=1$. We claim that for each $x_{0} \in X_{2} \backslash X_{1}$ there is a $\phi_{x_{0}} \in \operatorname{Hom}\left(\left[X_{1}+\left[A x_{0} B\right]\right], \mathscr{D}\right)$ which extends $\phi$ with the same cb-norm.

In fact, we may assume that $\left\|x_{0}\right\|=1$. Let $\mathscr{F}$ be the family of finite subset of $X_{1}$. Then $\mathscr{F}$ is a partial ordered space with the usual set-theoretic inclusion partial order. For each $F \in \mathscr{F},\left.\phi\right|_{[A F B]} \in \operatorname{Hom}([A F B], \mathscr{D})$. By the finite injectivity of $\mathscr{D}$, there is an extension $\phi_{x_{0}, F} \in \operatorname{Hom}\left(\left[A\left(F \cup\left\{x_{0}\right\}\right) B\right], \mathscr{D}\right)$ of $\left.\phi\right|_{[A F B]} \in \operatorname{Hom}([A F B], \mathscr{D})$ such that $\left\|\phi_{x_{0}, F}\right\|_{\mathrm{cb}}=\left\|\left.\phi\right|_{[A F B]}\right\|_{\mathrm{cb}}$. For each $F \in \mathscr{F}$, there is a subset $F_{x_{0}}$ of $\mathscr{D}$ consisting of all $y \in \mathscr{D}$ such that there is a $\psi \in \operatorname{Hom}\left(\left[A\left(F \cup\left\{x_{0}\right\}\right)\right], \mathscr{D}\right)$ which extends $\left.\phi\right|_{[A F B]}$ with the cb-norm less than or equal to 1 and such that $\psi\left(x_{0}\right)=y$. Then $F_{x_{0}}$ is a nonempty closed subset of the closed unit ball, ball $(\mathscr{D})$, of $\mathscr{D}$ which is compact in the weak operator topology. In fact, by the above argument, $F_{x_{0}} \neq \varnothing$ and $F_{x_{0}} \subseteq$ ball $(\mathscr{D})$ because $\|\psi\|_{c b} \leq 1$ and $\left\|x_{0}\right\|=1$. Suppose that $\left(y_{\lambda}\right)$ is a net in $F_{x_{0}}$ the converges to 
some $y$ in the weak operator topology. Since the ball $(\mathscr{D})$ is compact in the weak operator topology, $y \in \operatorname{ball}(\mathscr{D})$. Let $\phi_{\lambda} \in \operatorname{Hom}\left(\left[A\left(F \cup\left\{x_{0}\right\} B\right], \mathscr{D}\right)\right.$ be the extension of $\left.\phi\right|_{[A F B]}$ such that $\phi_{\lambda}\left(x_{0}\right)=y_{\lambda}$ and $\left\|\phi_{\lambda}\right\| \leq 1$. Since $\left.\phi_{\lambda}\right|_{[A F B]+A x_{0} B}$ is totally determined by $y_{\lambda}$, the limit $\psi=W-\left.\lim \phi_{\lambda}\right|_{[A F B]+A x_{0} B}$ exists. Since the cb-norm is lower semicontinuous in the weak operator topology, we have $\psi \in$ $\operatorname{Hom}\left([A F B]+A x_{0} B, \mathscr{D}\right)$ and $\|\psi\|_{\mathrm{cb}} \leq 1$. Since $[A F B]+A x_{0} B$ is norm dense in $\left[A\left(F \cup\left\{x_{0}\right\}\right) B\right], \psi$ may be uniquely continuously extended to $\left[A\left(F \cup\left\{x_{0}\right\}\right) B\right]$. Denoting the extension by $\psi$ also, we have $\psi \in \operatorname{Hom}\left(\left[A\left(F \cup\left\{x_{0}\right\}\right) B\right], \mathscr{D}\right)$, $\|\psi\|_{\mathrm{cb}} \leq 1$, and $\psi\left(x_{0}\right)=y$. Therefore, $y \in F_{x_{0}}$ and $F_{x_{0}}$ is closed in weak operator topology.

If $\left\{F_{i, x_{0}}, F_{i} \in \mathscr{F}, 1 \leq i \leq n\right\}, n \in \mathbf{N}$, is a finite subcollection of $\left\{F_{x_{0}}, F \in\right.$ $\mathscr{F}\}$, then $\bigcup F_{i} \in \mathscr{F}$ and $\left(\bigcup F_{i}\right)_{x_{0}} \subseteq F_{i, x_{0}}$ for all $1 \leq i \leq n$. Therefore, $\left\{F_{x_{0}}, F \in \mathscr{F}\right\}$ has finite intersection property. Since ball $(\mathscr{D})$ is compact in the weak operator topology, there is a $y_{0} \in \bigcap\left\{F_{x_{0}}, F \in \mathscr{F}\right\}$. Define $\phi_{x_{0}}: X_{1}+$ $A x_{0} B \rightarrow \mathscr{D}$ in the following way: for each $x \in X_{1}+A x_{0} B$, there is a $F \in \mathscr{F}$ such that $x \in\left[A\left(F \cup\left\{x_{0}\right\}\right) B\right]$; let $\phi_{F, x_{0}} \in \operatorname{Hom}\left(\left[A\left(F \cup\left\{x_{0}\right\}\right) B, \mathscr{D}\right)\right.$ be such that $\phi_{F, x_{0}}\left(x_{0}\right)=y_{0}$ and $\left\|\phi_{F, x_{0}}\right\| \leq 1$; and set $\phi_{x_{0}}(x)=\phi_{F, x_{0}}(x)$. That $\phi_{x_{0}}$ is a well-defined homomorphism that follows from the definition of $y_{0}$. Moreover, $\left\|\phi_{x_{0}}\right\|_{\mathrm{cb}}=\|\phi\|_{\mathrm{cb}}$ because $1=\|\phi\|_{\mathrm{cb}}=\sup \left\|\left.\phi\right|_{[A F B]}\right\|_{\mathrm{cb}}$. Since $X_{1}+A x_{0} B$ is dense in the $\left[X_{1}+\left[A x_{0} B\right]\right]$, we may continuously extend $\phi_{x_{0}}$ to $\left[X_{1}+\left[A x_{0} B\right]\right]$, obtaining $\phi_{x_{0}} \in \operatorname{Hom}\left(\left[X_{1}+\left[A x_{0} B\right]\right], \mathscr{D}\right)$ which extends $\phi$ with the same cbnorm.

Let $\mathscr{G}$ be the family of pairs $\left(\phi_{Y}, Y\right)$, where $Y$ is an $A-B$ operator subbimodule of $X_{2}$ containing $X_{1}$ and $\phi_{Y} \in \operatorname{Hom}(Y, \mathscr{D})$ which extends $\phi$ with the same cb-norm. By the argument just given, $\mathscr{G}$ is a nontrivial family. We give $\mathscr{G}$ the partial order defined by $\left(\phi_{Y_{1}}, Y_{1}\right) \preceq\left(\phi_{Y_{2}}, Y_{2}\right)$ if $Y_{1}$ is an $A-B$ operator sub-bimodule of $Y_{2}$ and $\left.\phi_{Y_{2}}\right|_{Y_{1}}=\phi_{Y_{1}}$. By Zorn's lemma, there is a maximal element $\left(\phi_{Y_{0}}, Y_{0}\right)$. From the initial step, we see that $Y_{0}=X_{2}$. Letting $\tilde{\phi}=\phi_{Y_{0}}$ yields the desired extension.

\section{REFERENCES}

1. William Arvesion, Subalgebras of $C^{*}$-algebra, Acta Math. 123 (1969), 141-224.

2. David P. Blecher, Zhong-Jin Ruan, and Allian M. Sinclair, A characterization of operator algebras, J. Funct. Anal. 89 (1990), 188-175.

3. Erik Christensen, Edward G. Effros, and Allan Sinclair, Completely bounded multilinear maps and $C^{*}$-algebras cohomology, Invent. Math. 90 (1987), 279-296.

4. Erik Christensen and Allan Sinclair, A survey of completely bounded operators, Bull. London Math. Soc. 21 (1989), 417-448.

5. Paul S. Muhly and Qiyuan Na, Extension of completely bounded $A-B$ bimodule maps, preprint, 1992.

6. _ Dilation of operator bimodules, preprint, 1992.

7. Vern I. Paulsen, Completely bounded maps and dilations, Pitman Res. Notes in Math. Ser., vol. 146, Longman, New York, 1986.

8. Anal. 55 (1984), 1-17.

9. Vern I. Paulsen and R. R. Smith, Multilinear maps and tensor norm on operator system, J. Funct. Anal. 73 (1987), 258-276.

10. Vern I. Paulsen and Ching Yun Suen, Commutant representation of completely bounded maps, J. Operator Theory 131 (1985), 87-101. 
11. A Guyan Robertson, Injective matrical Hilbert spaces, Math. Proc. Cambridge Philos. Soc. 110 (1991), 183-190.

12. Zhong-Jin Ruan, Subspaces of $C^{*}$-algebras, J. Funct. Anal. 76 (1988), 217-230.

13. __ Injectivity of operator spaces, Trans. Amer. Math. Soc. 315 (1989), 89-104.

14. R. R Smith, Completely bounded module maps and the Haagerup tensor product, J. Funct. Anal. 102 (1991), 156-175.

15. G. Wittstock, Extension of completely bounded $C^{*}$-module homomorphisms, Proc. Conf. On Operator Algebras and Group Representations (Neptun, 1980), Pitman, New York, 1983, pp. 238-250.

Department of Mathematics, University of lowa, Iowa City, Iowa 52242

E-mail address: nacoak.math.uiowa.edu 